\title{
Anisotropic properties of aligned SWNT modified poly (methyl methacrylate) nanocomposites
}

\author{
WEIXUE LI, QING WANG and JIANFENG DAI*, \\ Department of Physics, ${ }^{\dagger}$ State Key Laboratory of New Nonferrous Metal Materials, Lanzhou University of \\ Technology, Lanzhou 730050, P R China
}

MS received 15 July 2005

\begin{abstract}
The poly (methyl methacrylate) (PMMA)/single-walled carbon nanotube (SWNT) composites with good uniformity, dispersion and alignment of SWNT were fabricated in an improved figuration process. The semidried mixture was stretched along one direction at a drawing ratio of $\mathbf{5 0}$ before it was dried, and then folded along the same direction stretching repeatedly for 100 times. The transmission electron microscopic (TEM) observation demonstrated that SWNT in the PMMA/SWNT composite tends to align in the stretching direction owing to a torque exerting on it in the stretching process. The electrical and mechanical properties of PMMA/SWNT composite were studied as a function of SWNT orientation and concentration. The aligned SWNT modified PMMA/SWNT composite presented highly anisotropic properties. The experimental results showed that the electrical conductivity and mechanical properties of composite rise with the increase of SWNT concentration, and that composite films showed higher conductivity and higher mechanical draw ratios along the stretched direction than perpendicular to it. The thermogravimetric analysis (TGA) revealed that embedding the SWNTs into the PMMA matrix also improves the thermal stability of the composite.
\end{abstract}

Keywords. Single-walled carbon nanotube; poly methyl methacrylate; nanocomposite; anisotropic property.

\section{Introduction}

Since the discovery of single-walled carbon nanotubes (SWNT) in 1993, owing to the rapid development of synthesis methods for carbon nanotubes (CNTs), high quality, long and aligned CNT ropes are now available (Bethune et al 1993; Iijima and Ichihashi 1993). These advances in synthesis methods enabled the mechanical properties of CNTs to be more easily assessed. On account of their novel, structural, mechanical, and electronic properties, there is considerable interest in fabricating composite materials containing carbon nanotubes, both from the point of view of fundamental property determination and the potential applications in many fields. To date, some of the most important mechanical and electrical properties of polymer/CNT composites, such as the Young's modulus, tensile strength, and electric conductivity have been characterized experimentally (Ajayan et al 1994; Curran et al 1998; Jia et al 1999; Thostenson et al 2001; Allaoui et al 2002; Cooper et al 2002; Breur and Sundararaj 2004; Du et al 2004; Park et al 2004; Putz et al 2004).

Uniform dispersion and well alignment of CNTs within the polymer matrix, as well as improved matrix/nanotube wetting and adhesion are critical factors in the processing of these nanocomposites. Well alignment of CNT in nano-

*Author for correspondence (jfdai@MIT.EDU; daijf@lut.cn) composite is a more challenging work. Many of the preparation methods produce samples in which the CNTs are randomly oriented. Although the tubes are often grouped into bundles, the bundles themselves are not generally aligned with each other. In measuring CNT properties and field emission experiments, it would be very helpful to have samples in which all tubes were aligned in a specific direction. Some groups reported that the methods of aligning CNTs is to incorporate the tubes into a matrix and then extrude the matrix in some way, so that the CNTs become aligned along the direction of flow (Ajayan et al 1994; Curran et al 2004). Here we report the preparation of PMMA/SWNT composites with good uniformity and dispersion, which were fabricated in an improved process. Poly methyl methacrylate (PMMA) (weight-average molecular weight: $10000 \mathrm{~g} / \mathrm{mol}$ ) was chosen as matrix in this study for its good fibre spinning qualities and solubility in dimethylformamide (DMF). After being stretched at a large draw ratio along one direction, the SWCNT were well aligned along the stretched direction in the polymer matrix. The new materials were investigated by TEM, TGA, electrical analysis and mechanical test. The experimental results show that the mechanical properties and electrical conductivity of nanocomposite rise with the increase of SWNT loading. The aligned SWNT modified PMMA/SWNT composite presented highly anisotropic properties. Embedding the SWNTs into the PMMA matrix also improves the thermal stability of the composite. 


\section{Experimental}

Quasi-straight single-walled carbon nanotube (SWNT) bundles were efficiently prepared in large scale by anode-arc vapourization of graphite rod in helium atmosphere with metallic catalysts, by using our semicontinuous synthesis installation in this study. Owing to the pinching effect of arc current, the vapourized graphite sheet rolled up along the arc current, forming the quasi-straight SWNTs. The optimal conditions for the production of SWNT in our experiment were: a helium gas atmosphere of $6.0 \times 10^{4} \mathrm{~Pa}$, an arc current of $70 \mathrm{~A}$, a voltage drop of $24 \mathrm{~V}$, a constant distance $(\sim 2 \mathrm{~mm})$ between anodes and cathode, a metallic catalyst mixture powder of Fe-Co-Ni $(1.0 \mathrm{wt} \%, 1.0 \mathrm{wt} \%$, and $1.0 \mathrm{wt} \%$, respectively), and well cooled electrodes and collector.

To eliminate impurities in the pristine SWNT bundles such as metallic catalyst residues and amorphous carbon, they were treated in concentrated nitric acid at $80^{\circ} \mathrm{C}$ for $1 \mathrm{~h}$, followed by a reflux process in hydrochloric acid for $4 \mathrm{~h}$. After being washed, the treated SWNT bundles were obtained. The volume fraction of the impurity was estimated to be $\sim 5 \%$ by transmission electron microscopy (figure 1), and the weight content of impurity was measured as $7 \%$ using thermogravimetric analysis (TGA). The diameter of these SWNTs was specified as $1.2-1.5 \mathrm{~nm}$ and length, $>2 \mu \mathrm{m}$. It can be seen that the appearance is quite different to MWNT, the SWNTs were quasi-straight bundles rather than curled or entangled ones, where weak Van der Waals forces bind the tubes together. These acidtreatments are known to shorten the length of SWNT and introduce hydroxylic functional groups to CNT. It also makes SWNT bundles more dispersible in DMF.
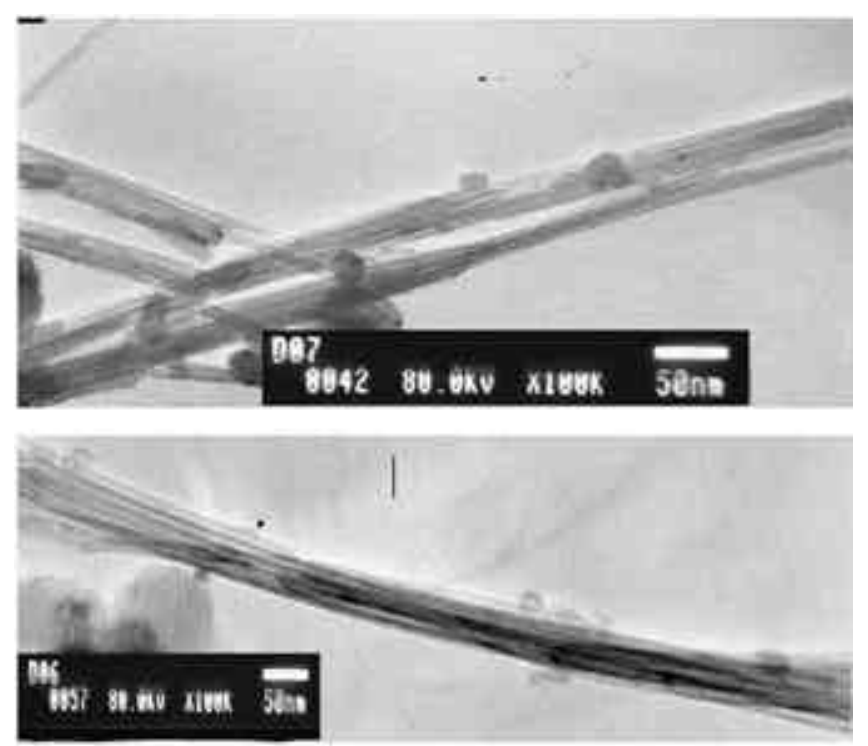

Figure 1. TEM micrographs of typical SWNT bundle dispersed in DMF.
The SWNT bundles were ground to fine powders and sonicated in DMF for $1 \mathrm{~h}$ at room temperature. PMMA was then dissolved into the nanotube/DMF suspension. After another hour of sonication, the suspension was air-dried in a fume hood for $1 \mathrm{~h}$. In order to align the SWNTs, the semidried mixture was stretched along one direction at a draw ratio of 50 before it was dried, and then folded along the same direction and stretched repeatedly for 100 times. In the stretching process, every SWNT in the matrix having an angular displacement tends to align in the stretching direction due to a torque exerting on it. Only when SWNT aligned along the stretching direction, the torque equalled zero. The mixed samples were then compressed under a pressure of about $10 \mathrm{MPa}$ at $200^{\circ} \mathrm{C}$ for 5 min using a hydraulic press. Finally, the matrix was transferred into a Teflon mold and air-dried in a fume hood overnight at $20^{\circ} \mathrm{C}$. The grayish and transparent composite films with nanotube weight fractions up to $10 \%$ were made via this wet-stretching and melt pressing process. For comparison with the composites, PMMA was dissolved in DMF and cast into a film following the same method as given above.

The cast film samples were cut into ultra-thin films of $\sim 50 \mathrm{~nm}$ thickness for TEM examination using a diamond knife. The TEM photograph (figure 2) demonstrated that

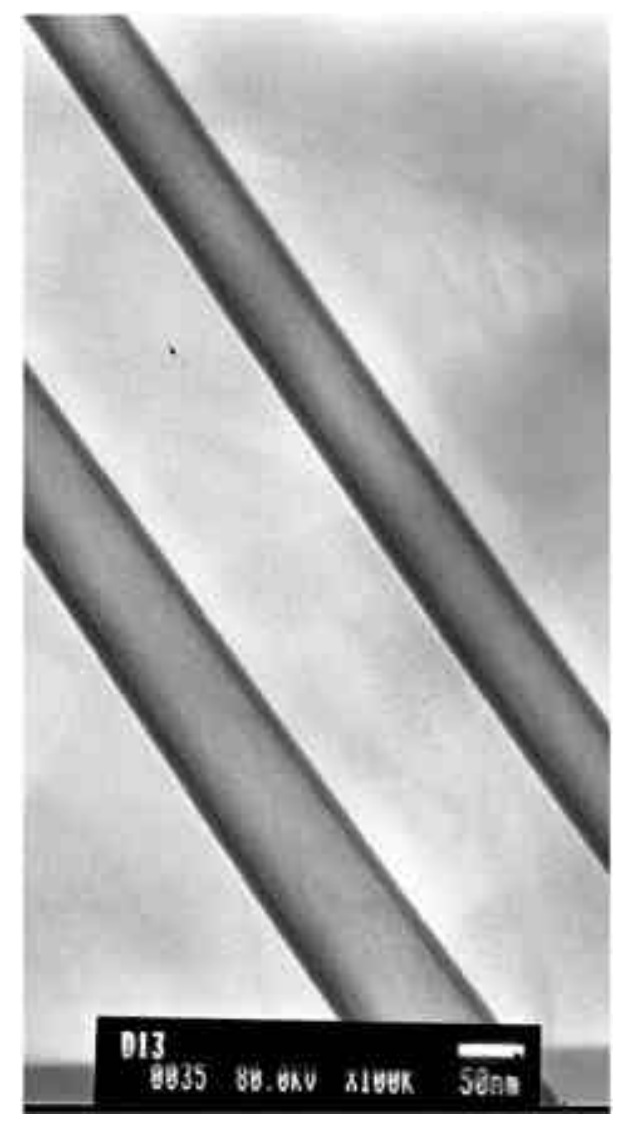

Figure 2. TEM micrograph of SWNT wrapped by PMMA aligning along the stretching direction in PMMA/SWNT composite. 
the SWCNTs aligned along the stretching direction and were wrapped up with PMMA of 60-100 nm diameters. It is suggestive of strong bonding between the nanotubes and the polymer matrix. Just as other groups reported, the SEM observation also shows good dispersion of SWNTs in PMMA and well alignment of SWNT parallel to the stretching direction.

In order to investigate the electrical properties of the nanocomposites, all the samples were cut on both sides to obtain flat and parallel surfaces. Rectangular specimens of about $50 \mathrm{~mm}$ length, $10 \mathrm{~mm}$ width, and $2 \mathrm{~mm}$ thickness were cut, and the cross section areas were painted with conductive silver to provide good contact with the specimen. The electrical measurement results were obtained from measurements of d.c. resistance between two terminals using a multimeter with a range of resistance from $20 \Omega$ $20 \mathrm{M} \Omega$.

Nanocomposite films with various SWNT weight fractions up to $8 \mathrm{wt}$.\% were made via the process described above and pure PMMA film was made by the same process for comparison. The films were cut into $3 \times 5 \mathrm{~mm}$ strips. The strips were heated at a temperature of $120^{\circ} \mathrm{C}$ that was slightly above the glass transition $\left(T_{\mathrm{g}}=115^{\circ} \mathrm{C}\right)$ of the polymer. Then, the strips were mechanically stretched up to their fracture by applying a constant force in the form of an attached weight. The maximum draw ratios (final length, $L$ over initial length, $L_{0}$ ), $\lambda_{\max }$, were obtained.

The samples were also characterized by thermogravimetric analysis (TGA) under a nitrogen atmosphere from room temperature to 700 at a heating rate of $10^{\circ} \mathrm{C} / \mathrm{min}$ using a TA Instruments SDT 2960.

\section{Results and discussion}

Figure 3 shows the volume electrical conductivity of PMMA/ SWNT composite as a function of loading. It indicated

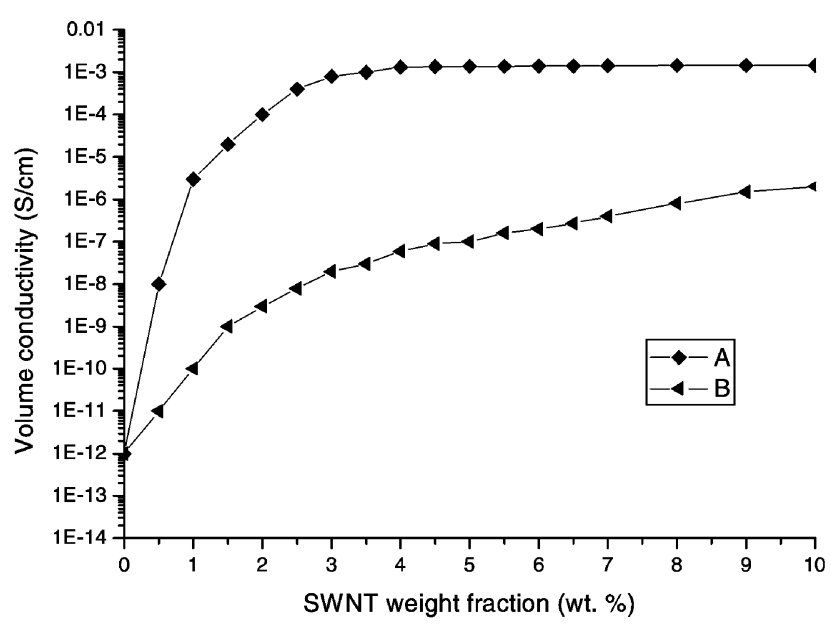

Figure 3. DC volume conductivity as a function of SWNT weight fraction of PMMA/SWNT film. (A) Along the alignment of SWNT and (B) perpendicular to the alignment of SWNT. that the electrical conductivity of PMMA/SWNT nanocomposite films increase substantially with SWNT composition. For example, the conductivity of the composites along the aligning direction of SWNT increased from $10^{-8}$ to $3 \times 10^{-6} \mathrm{~S} / \mathrm{cm}$ as the SWNTs composition increased from 0.5 wt. $\%-1.0$ wt.\% in PMMA, respectively. With respect to pure PMMA, the electrical conductivity of $3 \mathrm{wt} \%$ aligned nanocomposite increases by 9 orders of magnitude, up to $10^{-3} \mathrm{~S} / \mathrm{cm}$. This may be attributed to the doping effect associated with SWNTs which were believed to help induce the formation of a more efficient matrix for charge transport, thus enhancing the conductivity of the films. The PMMA/SWNT composite films showed higher conductivity along the aligning direction of SWNT than perpendicular to it. For example, for the same sample with a $1 \mathrm{wt} . \%$ SWNT loading, the electrical conductivity along the SWNT aligned direction was 4 orders of magnitude higher than that perpendicular to it. It is probably for this reason that SWNTs provide a conductive path along the aligning direction of SWNTs. Whereas, perpendicular to the aligning direction of SWNTs, PMMA separated the conductive path. However, our experimental result was different from Du et al's (2004) result on this point. They reported that the alignment of nanotubes in composite worsens the electrical conductivity by about 6 orders of magnitude. But, it was not clear how they obtained the experimental results. Though the conductivity increases with SWNT weight fractions both along and perpendicular to the aligning direction of SWNTs, but when the SWNT weight fraction in the PMMA matrix was higher than $3 \mathrm{wt} . \%$, the volume conductivity along the SWNT aligning direction could not increase with the SWNT loading any more. This indicates that aligned composite requires more nanotubes to reach the electrical conductivity threshold. This behaviour is indicative of a percolation transition. Percolation theory predicts that there is a critical concentration or percolation threshold at which a conductive path is formed in the composite causing the material to convert from a capacitor to a conductor.

The maximum draw ratios (final length, $L$ over initial length, $\left.L_{0}\right), \lambda_{\max }$, of the PMMA/SWNT composite and pure PMMA strips along and vertical to the stretching direction were presented in table 1 as a function of SWNT concentration. Just as the electrical conductivity results presented above, the PMMA/SWNT composite strips showed higher maximum draw ratios along the aligning direction of SWNT than perpendicular to it. It indicated that SWNTPMMA nanocomposite presents highly anisotropic properties when SWNTs aligned in the nanocomposite. Although the SWNTs were wrapped by PMMA and formed good interaction with PMMA, the maximum draw ratios of nanocomposite vertical to the SWNTs aligning direction were lower than that of along the aligning direction, even more, were lower than pure PMMA. On the one hand, the large weight fraction of SWNT usually lead to flocculation of SWNT and that is harmful to improve the 
Table 1. SWNT/PMMA composites and resulting maximum draw ratios, $\lambda_{\max }=L / L_{0}$.

\begin{tabular}{llcccccc}
\hline Materials & & Pure PMMA & 0.5 wt. $\%$ SWNTs & 1 wt. $\%$ SWNTs & 3 wt.\% SWNTs & 8 wt.\% SWNTs & 10 wt. $\%$ SWNTs \\
\hline$\lambda_{\max }$ & Parallel & 3500 & 3800 & 4000 & 5000 & 5500 & 6000 \\
& Perpendicular & 3000 & 2500 & 300 & 250 & 150 & 140 \\
\hline
\end{tabular}

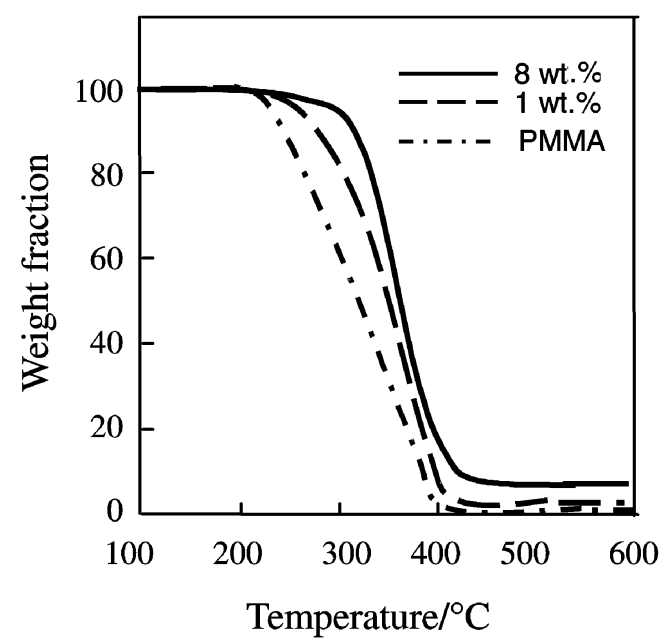

Figure 4. TGA of PMMA/SWNT composites and pure PMMA.

mechanical property of nanocomposite; on the other hand, the embedding of quasi-straight SWNTs introduce many dislocation lines into polymer matrix, and the dislocation motion usually forms cracks which worsen the mechanical property vertical to the SWNTs aligning direction. So, the SWNT improved the mechanical property of composite along the SWNTs aligning direction evidently, but worsened the mechanical property vertical to it.

The samples of pure PMMA, PMMA/SWNT composites with SWNT loading of $1 \%$ and $8 \%$ were also characterized by TGA in nitrogen at a heating rate of $5^{\circ} \mathrm{C} / \mathrm{min}$. Figure 4 shows the residual weight percentage vs temperature, and it also shows that pure PMMA and the nanocomposites with various SWNTs loading begin to lose weight at the same temperature, but the temperature at the maximum mass loss rate is $368^{\circ} \mathrm{C}$ for $1 \mathrm{wt} . \%$ SWNT composite, and $380^{\circ} \mathrm{C}$ for $8 \mathrm{wt} \%$ SWNT composite, which are $50^{\circ} \mathrm{C}$ and $62^{\circ} \mathrm{C}$ higher than that for PMMA, respectively. It indicated that embedding the SWNTs into the polymer matrix also improves the thermal stability of the polymer as reported by Du et al (2004).

\section{Conclusions}

Quasi-straight single-walled carbon nanotube (SWNT) bundles have been efficiently prepared in quantum (grams) by anode-arc vapourization of graphite rod with metallic catalysts in helium atmosphere. The PMMA/SWNT composites with good uniformity and dispersion were fabricated in an improved in situ process. After being stretched at a large draw ratio along one direction, the SWCNT were well aligned along the stretched direction in the polymer matrix owing to a torque exerting on it in the stretching process. The TEM photograph demonstrated that the SWCNTs aligned along the stretching direction and were wrapped up with PMMA of 60-100 nm diameter. The experimental results show that the mechanical properties and electrical conductivity of the PMMA/SWNT nanocomposite rise with the increase of SWNT. With respect to pure PMMA, the electrical conductivity of $3 \mathrm{wt} . \%$ aligned SWNT nanocomposite increased by 9 orders of magnitude, up to $10^{-3} \mathrm{~S} / \mathrm{cm}$, and the maximum draw ratio was higher up to 5000 than pure PMMA of 3500 . The aligned SWNT modified PMMA/SWNT composite presented highly anisotropic properties, as a result the composite films showed higher conductivity and higher mechanical draw ratios along the stretched direction than perpendicular to it. The PMMA/SWNT composites were provided with enhanced mechanical and electrical properties and exceptional nanotube alignment as well. TGA revealed that embedding the SWNTs into the PMMA matrix also improves the thermal stability of the composite.

\section{Acknowledgements}

This work was financially supported by Natural Science Foundation of Gansu Province and the Tackle Key Problems Foundation of Gansu Province, People's Republic of China.

\section{References}

Ajayan P M, Stephan O, Colliex C and Trauth D 1994 Science 2651212

Allaoui A, Bai S, Cheng H M and Bai J B 2002 Compos. Sci. \& Technol. 6262

Bethune D S, Kiang C H, de Vries M S, Gorman G, Savoy R, Vasquez J and Beyers R 1993 Nature 363605

Breuer O and Sundararaj U 2004 Polym. Comp. 25630

Cooper C A, Ravich D, Lips D, Mayer J and Wagner H D 2002 Compos. Sci. \& Technol. 621105

Curran S A, Ajayan P M, Blau W J, Carroll D L, Coleman J N, Dalton A B and Davey A P 1998 Adv. Mater. 101091

Du F, Fischer J E and Winey K I 2004 J. Polym. Sci. B41 3333

Iijima S and Ichihashi T 1993 Nature 363603

Jia Z, Wang Z, Xu C, Liang J, Wei B, Wu D and Zhu S 1999 Mater. Sci. \& Eng. A271 395

Park S J, Cho M S, Lim S T, Choi H J and Jhon M S 2004 Macromol. Rapid Commun. 241070

Putz K W, Mitchell C A, Krishnamoorti R and Green P F 2004 J. Polym. Sci. B42 2286

Thostenson E T, Ren Z and Chou T W 2001 Compos. Sci. \& Technol. 61899 